\title{
Níveis de aptidão física e perfil antropométrico dos árbitros de elite do Paraná credenciados pela Confederação Brasileira de Futebol (CBF)
}

\author{
Alberto I. Silva ${ }^{1}$ \\ Ciro Rodriguez-Añez ${ }^{2}$
}

https://doi.org/10.5628/rpcd.03.03.18

\author{
${ }^{1}$ Unidade de Ensino Superior do Vale do Iguaçu \\ Uniguaçu, Brasil, \\ ${ }^{2}$ Pontifícia Universidade Católica do Paraná, Brasil.
}

\section{RESUMO}

Este estudo teve como objetivo avaliar o nível de aptidão física e a composição corporal dos árbitros de elite da Federação Paranaense de Futebol (FPF). A amostra foi constituída por 16 árbitros que se submeteram a provas de aptidão física na temporada de 2000. Todos os indivíduos eram do sexo masculino, apresentavam uma idade média de $34,5 \pm 4,8$ anos, estatura de $177,8 \pm 7,4 \mathrm{~cm}$ e massa corporal de $78,7 \pm 8,0 \mathrm{~kg}$. A bateria de testes utilizada para avaliar a condição física foi a padronizada pela FIFA, e inclui uma prova de corrida de 50 metros, outra de 200 metros e o teste de Cooper. Para avaliação da composição corporal foram mensuradas 9 dobras cutâneas (subescapular, tríceps, bíceps, peitoral, axilar média, abdómen, suprailíaca, coxa e panturrilha), 9 perímetros (antebraço, braço contraído, braço relaxado, tórax, abdómen, quadril, coxa superior, coxa média e panturrilha), e 4 diâmetros ósseos (biestilóide, biepicondiliano, bicondiliano e bimaleolar) $(17,18,19)$. A partir das variáveis antropométricas determinou-se a densidade corporal conforme equação de Jackson e Pollock (20) e o percentual de gordura, utilizando-se a equação de Siri (21). O peso da gordura (PG) foi obtido multiplicando-se a massa corporal (MC) pela fração do percentual de gordura $(\% \mathrm{G}), \mathrm{PG}=\mathrm{MC}(\% \mathrm{G} / 100)$. Para a determinação do peso ósseo (PO) e do peso residual (PR) foram utilizadas, respectivamente, as equações de Von Döblen e Würch citados por De Rose et. al. (22). O peso muscular foi obtido subtraindo-se da MC o PO, PR e PG. Os resultados dos testes são reportados como valores médios com o respectivo desvio-padrão e/ou erro-padrão. Comparações entre valores médios foram realizadas pela análise de variância (ANOVA). A comparação entre as idades dos grupos foi realizada pelo teste não paramétrico de Mann-Withtney-U. No estudo da correlação foi utilizado o coeficiente de Pearson. Significâncias estatísticas foram consideradas quando $p \leq 0,05$. Após a aplicação da bateria de testes padronizada pela FIFA, foi possível observar que em média o árbitro paranaense demora $6,81 \pm 0,31 \mathrm{~s}$ a percorrer os 50 metros e $28,85 \pm 1,57 \mathrm{~s}$ a percorrer os 200 metros. Estes valores são, em ambas as provas, melhores que os obtidos pelos árbitros que participaram no concurso para novos árbitros da FIFA em 1995 (15). O valor médio da corrida de 12 minutos foi de $2956 \pm 90,69$ metros. Os dados antropométricos demonstram que os árbitros do nosso estudo possuem valores de estatura, massa corporal e componentes da composição corporal maiores do que os de árbitros de outros estudos, porém uma massa de gordura menor.

\author{
ABSTRACT \\ Fitness levels and anthropometric profile of elite referees of \\ Paraná certified by the Brazilian Football Confederation (CBF)
}

The aim of this study was to evaluate the levels of physical fitness and body composition of elite referees from the Football Federation of Paraná (FPF). Sample size comprises 16 referees from the FPF, all males, that belong to the national board (certified by the Brazilian Football Confederation). Fitness evaluation was applied during the 2000 Soccer Season. Mean age was $34.5 \pm 4.8$ years, height was $177.8 \pm 7.4 \mathrm{~cm}$, and body weight was $78.7 \pm 8.0 \mathrm{~kg}$. The FIFA's standardized test battery includes the following tests: a 50 meters and a 200 meters running test and the Cooper test. To assess body composition we measured skinfolds (subscapular, triceps, biceps, pectoral, medium axillary, abdomen, suprailiac, thigh and calf), girths (forearm, contracted arm, relaxed arm, thorax, abdomen, hip, thigh, mid-thigh and calf) and breadths (biacromial, biiliocristal, biepicondylar humerus and maleolar) $(17,18,19)$. From anthropometrical variables, body density was determined according to Jackson and Pollock's (20) equation and the fat percentual using Siri's (21) equation. Fat weight (PG) was calculated multiplying the body mass $(M C)$ by the fraction of fat percentual (\% G), $P G=M C(\% G / 100)$. Bone weight $(P O)$ and residual weight $(P R)$ were estimated using respectively Von Döblen's and Würch's equations mentioned in De Rose et. al (22). Muscular weight (PM) was obtained by subtracting to MC the PO, PR and PG. Tests results are ascribed as mean values ( \pm standard deviation) and/or standard error of the mean. Comparisons between mean values were performed with analysis of variance (ANOVA). Comparison between age groups was performed with Mann-Withney-U test. In the correlation study Pearson's $r$ was used. Statistical significances were considered when $p \leq 0,05$. Results showed that the referees from Paraná, Brazil, cover the 50 meter running test in $6.81 \pm 0.31 \mathrm{~s}$., and the average time for the 200 meter running test is $28.85 \pm 1.57 \mathrm{~s}$. These values are, for both running distances, better than those of the referees who apply to FIFA referee in 1995(15). Mean distance covered in the Cooper test was $2956 \pm 90.69$ meters. Anthropometric data show that the referees in our study have higher values of height, body mass, and body composition components than referees in other studies. However they have a lower fat mass.

Key Words: referee, soccer, physical fitness, tests. 


\section{INTRODUÇÃO}

Em 1868, numa das várias modificações que o futebol sofreu ao longo da sua história, surge o árbitro (1). Contudo, é somente em 1881 que esta figura tão importante para o futebol aparece definitivamente (2). Nos primórdios, o árbitro dirigia as partidas de futebol sem uma regra que estipulasse quais eram seus direitos e deveres, só intervindo na jogada quando alguém de uma das equipes reclamasse. Em 1890 é regulamentada a sua função em campo, e em 1891 verifica-se uma revisão completa do código, passando o árbitro a ter dois assistentes que, ao contrário do árbitro, surgem com funções determinadas (2). No início, o árbitro não utilizava apito para interromper uma jogada (2). Quando entendia ter sido cometida uma falta, ele gritava para que os jogadores parassem. O apito somente começou a ser usado em 1878 no "Nottingham Forest Ground" (3).

O árbitro é tão importante para o futebol, que sem ele não pode ocorrer uma partida (4). Na realidade, para uma partida ser conduzida com eficiência deverão estar presentes no campo de jogo no mínimo três árbitros. Um atua como árbitro principal (aquele que apita a partida) e os outros dois atuam como árbitros assistentes, sendo conhecidos popularmente como bandeirinhas.

Durante muito tempo o árbitro de futebol foi considerado uma figura secundária. Hoje, o seu papel é tão importante, que uma decisão equivocada pode retirar um campeonato a uma equipe que investiu milhões de dólares na compra e preparação de jogadores. Devido à importância do árbitro, a comunidade cientifica passou a considerá-lo como objecto de investigação para melhor compreender a sua intervenção e fundamentar a sua preparação. Entre os trabalhos mais recentes encontram-se os que descrevem as ações motoras do árbitro de futebol durante a partida, as distâncias percorridas por ele $(5,6,7)$ e pelos árbitros assistentes (8), os parâmetros antropométricos, a acuidade visual, o condicionamento físico (9), e os parâmetros relacionados com a intensidade da atividade física do árbitro e do árbitro assistente durante a partida, mensurada pela freqüência cardíaca $(10,11)$. Estes trabalhos contribuem para a formulação de um treinamento especifico do árbitro de futebol.

Visando melhorar o rendimento dos árbitros, o treinamento físico deverá ser cada vez mais específico.
Para isto, faz-se necessário conhecer o maior número de variáveis que possam auxiliar na elaboração de programas de treinamento, que transfiram melhor seus efeitos para o esporte que se está treinando. A avaliação é um importante recurso que possibilita conhecer a situação e o desenvolvimento de determinado sistema energético ou de outras variáveis que influenciam o desempenho do árbitro, sendo a principal forma de retroalimentação do programa (12). A avaliação constitui-se assim como um meio e não como um fim em si mesma. Contudo, a avaliação de qualquer processo é uma tarefa muito difícil, pois requer conceitos bem definidos. Os testes de aptidão física diferenciam-se dos exames médicos, porque estes últimos servem para diagnosticar a condição de saúde ou doença de um indivíduo e para prever seu risco para desenvolver determinada patologia, enquanto que as avaliações de aptidão física servem para classificar as pessoas segundo sua aptidão física (13). O uso destes testes pelos profissionais de Educação Física pode ser justificado dos pontos de vista pedagógico e psicológico, já que os resultados permitem a avaliação objetiva de qualquer progresso (14). Os resultados de uma bateria de testes servem para verificar o potencial e as debilidades de um atleta, determinando assim a sua condição preliminar, durante e após o treinamento; permitem verificar se o programa está alcançando os objetivos traçados; e, finalmente, concluir quanto o atleta ganhou com o treinamento. Além de servirem de apoio ao diagnóstico do nível de rendimento do atleta, estes dados podem ser empregados para estimular o seu interesse pelo treinamento $(13,14,15)$.

Assim como houve uma grande evolução no treinamento esportivo, ocorreu também uma grande evolução nas formas de medir os resultados obtidos durante um programa de treinamento. "A medida é uma determinação de grandeza que se constitui no primeiro instrumento para obter informação sobre algum dado pesquisado" (12). As medidas objetivas como a força e a velocidade são simples e diretas, produzindo freqüentemente resultados mais confiáveis do que aquelas que envolvem a personalidade, a inteligência e as atitudes (12).

Poucos trabalhos falam das necessidades físicas, do perfil antropométrico e da composição corporal de árbitros de futebol. Para estabelecer programas espe- 
cíficos de treinamento é necessário conhecer além das exigências físicas da atividade, o perfil antropométrico do atleta para saber se este está além ou aquém do perfil adequado para a função (16). Sabese ainda que quantidades elevadas de gordura corporal prejudicam o desempenho dos indivíduos, além de constituirem fator de risco para diversas doenças, sendo portanto fundamental o controle adequado da adiposidade corporal.

Observa-se a necessidade de se investir em pesquisa para a melhora da performance física do árbitro de futebol evitando, desta maneira, que decisões equivocadas provocadas pelo esgotamento físico durante a partida tirem o brilho de um bom jogo de futebol. O objetivo deste trabalho foi caracterizar os níveis de aptidão física e o perfil antropométrico dos árbitros paranaenses pertencentes ao quadro da Confederação Brasileira de Futebol (CBF).

\section{MATERIAL E MÉTODOS}

A população deste estudo foi constituída por árbitros da Federação Paranaense de Futebol (FPF) que pertencem ao quadro da Confederação Brasileira de Futebol (CBF). Estes árbitros apresentaram-se à Comissão de Avaliação da Aptidão Física da FPF para serem submetidos às provas de aptidão física durante a temporada de 2000. A amostra foi constituída por 16 árbitros, todos do sexo masculino, com uma idade média de $34,5 \pm 4,8$ anos (tabela 1), estatura média de $177,8 \pm 7,4 \mathrm{~cm}$, e massa corporal média de $78,7 \pm 8,0 \mathrm{~kg}$. O número total de indivíduos avaliados correspondeu a $100 \%$ da população dos árbitros credenciados pela CBF no Paraná. Os árbitros foram divididos em dois grupos, integrando cada grupo 8 indivíduos. Um grupo integrava os indivíduos que estavam credenciados para atuar como árbitros e o outro os árbitros assistentes.

Tabela 1 - Média e mediana das idades dos árbitros e assistentes da CBF

\begin{tabular}{lcccccc} 
Idade & N & Média & Mediana* & Mínimo & Máximo & $\begin{array}{r}\text { Média } \\
\text { Geral }\end{array}$ \\
\hline Árbitros & 8 & 36,11 & 37,15 & 25,25 & 44,63 & N 16 \\
Assistentes & 8 & 32,93 & 33,92 & 29,18 & 35,17 & 34,5
\end{tabular}

Não há diferença entre as idades dos árbitros e assistentes da CBF (Teste de Mann-Withtney-U; $U=20 ; Z=1,26 ; p=0,207 ; N 1=N 2=8$ ]
As provas utilizadas foram as recomendadas pela FIFA, até a presente data, para avaliação da aptidão física de árbitros de futebol. A bateria de testes foi constituída por: 2 piques de 50 metros, 2 piques de 200 metros e uma corrida de 12 minutos (teste de Cooper). Estas provas foram aplicadas na seguinte ordem: primeiramente, 2 piques de 50 metros; depois, 2 piques de 200 metros, seguidos da corrida de 12 minutos. O tempo de recuperação durante as provas não foi inferior a 5 minutos. Os testes foram aplicados em uma pista de atletismo. Após a realização dos mesmos os sujeitos permaneciam caminhando até o local da próxima prova. Após a execução dos testes de velocidade foi dado um intervalo de 15 minutos antes da realização do teste de Cooper. Para mensuração dos tempos em cada uma das provas foram utilizados cronômetros da marca Technos, modelo Cronus.

Todas as provas são eliminatórias, isto é, o árbitro que reprova em qualquer prova não pode continuar a avaliação. O tempo máximo estabelecido pela FIFA para a corrida de 50 metros é de 7,50 segundos e para a corrida de 200 metros, é de 32 segundos. A distância mínima estabelecida para a corrida de 12 minutos é de 2700 metros. O árbitro que, por exemplo, não obtivesse o índice mínimo na primeira corrida de 50 metros, era eliminado dos testes. As provas foram realizadas no período matutino.

Para avaliação da composição corporal foram mensuradas 9 dobras cutâneas (subescapular, tríceps, bíceps, peitoral, axilar média, abdómen, suprailíaca, coxa e panturrilha), 9 perímetros (antebraço, braço contraído, braço relaxado, tórax, abdómen, quadril, coxa superior, coxa média e panturrilha), e 4 diâmetros ósseos (biestilóide, biepicondiliano, bicondiliano e bimaleolar) $(17,18,19)$. A massa corporal, estatura e idade também foram determinadas. A partir das variáveis antropométricas determinou-se a densidade corporal conforme equação de Jackson e Pollock (20) e o percentual de gordura, utilizando-se a equação de Siri (21). O peso da gordura (PG) foi obtido multiplicando-se a massa corporal (MC) pela fração do percentual de gordura $(\% \mathrm{G})$, $\mathrm{PG}=\mathrm{MC}(\% \mathrm{G} / 100)$. Para a determinação do peso ósseo (PO) e do peso residual (PR) foram utilizadas, respectivamente, as equações de Von Döblen e Würch citados por De Rose et. al. (22). O peso mus- 
cular foi obtido subtraindo-se da MC o PO, PR e PG. Os resultados dos testes são reportados como valores médios com o respectivo desvio-padrão e/ou erropadrão. Comparações entre valores médios foram realizadas pela analise de variância (ANOVA) modelo inteiramente casualisado. A comparação entre as idades dos grupos foi realizada pelo teste não paramétrico de Mann-Withtney-U. Significâncias estatísticas foram consideradas quando $\mathrm{p} \leq 0,05$. Para o teste de correlação foi utilizado o teste de Pearson, r, sendo que um valor de $\mathrm{p} \leq 0,05$ foi considerado significativo.

\section{RESULTADOS}

Os resultados obtidos pelos árbitros e pelos árbitros assistentes durante as provas de corrida de 50 metros estão listados na Tabela 2 . Os resultados do teste de velocidade aos 200 metros e do teste de Cooper são apresentados nas Tabelas 3 e 4 . Os resultados apresentados na Tabela 3 são apenas os relativos aos árbitros, pois, de acordo com as normas da $\mathrm{CBF}$, os assistentes não realizam esta prova. $\mathrm{Na}$ Tabela 5 são apresentados os resultados referentes às mensurações das variáveis antropométricas e na Tabela 6 as correlações entre as variáveis da composição corporal e os testes motores.

Tabela 2 - Duração (em segundos) dos piques de 50 metros dos árbitros e árbitros assistentes da CBF.

\begin{tabular}{llccc}
\multicolumn{5}{c}{ Piques } \\
\hline Árbitros & Média & $6,74(6,03-7,28)$ & $6,74(6,27 \cdot 7,24)$ & 6,74 \\
& dp & 0,43 & 0,35 & 0,38 \\
& $\mathrm{~N}$ & 8 & 8 & 16 \\
\hline Assistente & Média & $6,89(6,09-7,29)$ & $6,86(6,53-7,12)$ & 6,87 \\
& dp & 0,35 & 0,19 & 0,27 \\
& $\mathrm{~N}$ & 8 & 8 & 16 \\
\hline Piques & Média & 6,81 & 6,80 & \\
& $\mathrm{dp}$ & 0,39 & 0,28 & \\
& $\mathrm{~N}$ & 16 & 16 &
\end{tabular}

Não se encontraram diferenças estatisticamente significativas entre os resultados dos árbitros e dos árbitros assistentes. Também não foram encontradas diferenças de resultados com significado estatístico entre piques (Anova, $p>0,05$ ).

$N$ - número de dados; dp-desvio-padrão.
Tabela 3 - Duração (em segundos) dos piques em 200 metros dos árbitros da CBF.

\begin{tabular}{lccc} 
& N & Média & dp \\
\hline Pique 1 & 8 & $28,10(24,57-30,31)$ & 1,66 \\
Pique 2 & 8 & $29,59(26,12-32,84)$ & 1,90 \\
& & \\
Não se verificaram diferenças estatisticamente significativas & entre as médias dos piques (Anova, p>0,05) \\
N-número de dados; dp - desvio-padrão.
\end{tabular}

Tabela 4 - Distância percorrida no teste de Cooper pelos árbitros e pelos assistentes da CBF.

\begin{tabular}{lccc} 
& N & Média & dp \\
\hline Árbitros & 8 & $2883,75(2700-3090)$ & 147,84 \\
Assistentes & 8 & $3030,00(2830-3150)$ & 96,95
\end{tabular}

Foram encontradas diferenças estatisticamente significativas entre os resultados dos árbitros e os resultados dos assistentes (Anova $p<0,05$ ] $N$ - número de dados; dp-desvio-padrão. 
Tabela 5 - Estatística descritiva para as avaliações antropométricas dos árbitros e assistentes da CBF.

\begin{tabular}{|c|c|c|c|c|c|c|c|c|c|}
\hline \multirow[b]{2}{*}{ Estatura } & \multirow[b]{2}{*}{ Árbitros } & \multirow{2}{*}{$\frac{N}{8}$} & \multirow{2}{*}{$\begin{array}{l}\text { Média* } \\
176,69\end{array}$} & \multicolumn{2}{|c|}{$\begin{array}{c}\text { Lim. Confiança } \\
\text { [95\%] }\end{array}$} & \multirow{2}{*}{$\begin{array}{l}\text { Mínimo } \\
169,50\end{array}$} & \multirow{2}{*}{$\begin{array}{l}\text { Máximo } \\
195,00\end{array}$} & \multirow{2}{*}{$\frac{D p}{8,16}$} & \multirow{2}{*}{$\begin{array}{l}\text { Epm } \\
2,88\end{array}$} \\
\hline & & & & 169,87 & 183,51 & & & & \\
\hline & Assistentes & 8 & 178,88 & 172,66 & 185,09 & 170,00 & 190,00 & $?, 43$ & 2,63 \\
\hline \multirow[t]{2}{*}{ Peso } & Árbitros & 8 & 79,14 & 72,08 & 86,20 & 69,30 & 97,30 & 8,44 & 2,99 \\
\hline & Assistentes & 8 & 78,18 & 71,04 & 85,31 & 65,00 & 87,80 & 8,53 & 3,02 \\
\hline \multirow[t]{2}{*}{$\%$ Gordura } & Árbitros & 8 & 17,28 & 14,01 & 20,54 & 9,62 & 23,20 & 3,90 & 1,38 \\
\hline & Assistentes & 8 & 14,44 & 11,98 & 16,90 & 9,93 & 18,80 & 2,94 & 1,04 \\
\hline \multirow[t]{2}{*}{ Peso Gordura } & Árbitros & 8 & 13,80 & $10,5 ?$ & 17,02 & 6,93 & 18,61 & 3,85 & 1,36 \\
\hline & Assistentes & 8 & 11,39 & 8,91 & 13,87 & 6,45 & 15,66 & 2,97 & 1,05 \\
\hline \multirow[t]{2}{*}{ Peso Residual } & Árbitros & 8 & 12,89 & 11,56 & 14,23 & 11,15 & 16,33 & 1,60 & 0,56 \\
\hline & Assistentes & 8 & 13,63 & 12,06 & 15,19 & 11,18 & 16,12 & 1,87 & 0,66 \\
\hline \multirow[t]{2}{*}{ Peso Muscular } & Árbitros & 8 & $19,0 ?$ & 17,37 & 20,77 & 16,70 & 23,45 & 2,04 & 0,72 \\
\hline & Assistentes & 8 & 18,84 & 17,12 & 20,56 & 15,67 & 21,16 & 2,06 & 0,73 \\
\hline \multirow[t]{2}{*}{ PM } & Árbitros & 8 & 33,38 & 30,68 & 36,07 & 29,21 & 39,14 & 3,22 & 1,14 \\
\hline & Assistentes & 8 & 34,32 & 31,66 & 36,97 & 30,33 & 39,69 & 3,17 & 1,12 \\
\hline
\end{tabular}

*Não há diferenças estatisticamente significativas entre os valores médios dos árbitros e dos assistentes (Anova p>0,05]. dp - desvio-padrão; epm - erro padrão da média.

Tabela 6 - Correlações entre as variáveis da composição corporal e os testes motores.

\begin{tabular}{|c|c|c|c|c|c|c|c|c|c|c|}
\hline & $\% G$ & $P G$ & P0 & PR & PM & t50 1 & $\mathrm{t} 502$ & t200 1 & t2002 & Cooper \\
\hline \multirow[t]{2}{*}{$\% G$} & 1,0000 & ,9304 & ,0287 & ,4257 & -,2801 & ,2143 & ,0938 &, 5571 & 6507 &,- 6170 \\
\hline & $p=\cdots$ & $p=, 001$ & $p=, 946$ & $p=, 293$ & $\mathrm{p}=, 502$ & $p=, 610$ & $\mathrm{p}=, 825$ & $p=, 151$ & $\mathrm{p}=, 081$ & $p=, 103$ \\
\hline \multirow[t]{2}{*}{$P G$} & & 1,0000 & ,3560 & ,7265 & ,0728 & ,0987 & ,0492 & ,4343 &, 5230 &,- 6892 \\
\hline & & $p=\cdots$ & $p=, 387$ & $p=, 041$ & $\mathrm{p}=, 864$ & $p=, 816$ & $p=, 908$ & $p=, 282$ & $p=, 184$ & $p=, 059$ \\
\hline \multirow[t]{2}{*}{ PO } & & & 1,0000 & ,8106 & ,6912 &,- 1995 &,- 1368 & -,2298 &,- 4185 &,- 0408 \\
\hline & & & $p=\cdots$ & $p=, 015$ & $p=, 058$ & $p=, 636$ & $p=, 747$ & $p=, 584$ & $p=, 302$ & $p=, 924$ \\
\hline \multirow[t]{2}{*}{ PR } & & & & 1,0000 &, 7187 &,- 0920 & ,015? & ,0887 & ,1119 &,- 5618 \\
\hline & & & & $p=--\cdot$ & $p=, 045$ & $p=, 828$ & $p=, 971$ & $p=, 835$ & $p=, 792$ & $p=, 147$ \\
\hline \multirow[t]{2}{*}{ PM } & & & & & 1,0000 &,- 2022 & ,0402 &,- 2292 &,- 1955 &,- 2730 \\
\hline & & & & & $p=\cdots$ & $p=, 631$ & $p=, 925$ & $p=, 585$ & $p=, 643$ & $p=, 513$ \\
\hline \multirow[t]{2}{*}{ t50 1} & & & & & & 1,0000 & ,8728 & ,8065 &, $346 ?$ &,- 0164 \\
\hline & & & & & & $p=\cdots$ & $p=, 005$ & $p=, 016$ & $\mathrm{p}=, 400$ & $p=, 969$ \\
\hline \multirow[t]{2}{*}{ t502 } & & & & & & & 1,0000 & ,8380 &, 4753 & ,0062 \\
\hline & & & & & & & $p=\cdots$ & $p=, 009$ & $p=, 234$ & $p=, 988$ \\
\hline \multirow[t]{2}{*}{ t200 1} & & & & & & & & 1,0000 & ,7845 &,- 2236 \\
\hline & & & & & & & & $p=---$ & $p=, 021$ & $p=, 594$ \\
\hline \multirow[t]{2}{*}{ t2002 } & & & & & & & & & 1,0000 &,- 5413 \\
\hline & & & & & & & & & $p=\cdots$ & $p=, 166$ \\
\hline \multirow[t]{2}{*}{ Cooper } & & & & & & & & & & 1,0000 \\
\hline & & & & & & & & & & $p=-\cdot$ \\
\hline
\end{tabular}




\section{DISCUSSÃO}

O primeiro teste aplicado aos árbitros foi o teste de corrida de 50 metros. Para esta prova, o tempo máximo é de 7,50 segundos. O tempo médio dos árbitros foi de $6,81 \pm 0,31 \mathrm{~s}(\mathrm{n}=16)$. Este valor é inferior a média de 7,09 s obtida por 52 árbitros que participaram do concurso da União Europeia de Futebol "Association" (UEFA) para novos árbitros da FIFA, em 1995 (15). Os árbitros da UEFA percorreram em média o primeiro pique de 50 metros em $7,12 \mathrm{~s}$ e o segundo em 7,07 s. Os árbitros paranaenses apresentaram um tempo médio muito inferior a estes, nos dois piques (Tabela 2). Quando se compararam os tempos obtidos pelos árbitros paranaenses no primeiro e no segundo piques de 50 metros, não foram observadas diferenças estatisticamente significativas ( $p>0,05)$.

Quando comparados os tempos dos árbitros e dos árbitros assistentes na prova de 50 metros, constatou-se que os árbitros eram ligeiramente mais rápidos (Tabela 2). Contudo estas diferenças não se revelaram estatisticamente significativas $(p>0,05)$. Um bom tempo conseguido pelos árbitros no teste de 50 metros é muito importante, pois esta condição permitirá que o árbitro esteja mais próximo da bola no momento em que tenha que analisar uma jogada. O árbitro de futebol percorre uma distância superior a $9 \mathrm{~km}$ durante o jogo $(5,6,7,23,24,25)$, em uma área média de $8.250 \mathrm{~m}^{2}$. Já o árbitro assistente desloca-se em média 6.912 metros durante uma partida (8), numa linha reta com um comprimento de cerca de 60 metros. Como o desgaste físico do árbitro durante uma partida é superior ao do árbitro assistente, espera-se do árbitro principal melhores resultados durante os testes, pois um dos principais fatores para uma boa arbitragem é a preparação física $(5,15,25,26)$.

A segunda prova da bateria de testes da FIFA é a corrida de 200 metros. Esta prova só é realizada pelos árbitros principais da CBF, isto é, os árbitros que são indicados para atuar como árbitros assistentes não necessitam fazer esta prova. Esta prova possui características de uma prova anaeróbica láctica, isto porque, durante a sua realização, há um aumento do nível do lactato sangüíneo.

O tempo médio para percorrer os 200 metros pelos árbitros foi de $28,85 \pm 1,57$ segundos $(n=8)$. Este tempo é inferior ao tempo realizado pelos árbitros que participaram do concurso da UEFA. Nestes últi- mos, o tempo médio na prova de 200 metros foi de $29,08 \mathrm{~s}$ para o primeiro pique e de $29,26 \mathrm{~s}$ para o segundo (15). Os árbitros paranaenses, apesar de terem um tempo médio menor nesta prova, apresentaram uma diferença muito maior entre o tempo obtido na primeira prova de 200 metros e o tempo obtido na segunda prova (Tabela 3). Diferentemente aos piques de 50 metros, o tempo na corrida de 200 metros aumentou da primeira para a segunda corrida, embora sem significado estatístico.

O teste de Cooper é o último da bateria de testes da FIFA e consiste em correr ou andar ininterruptamente durante 12 minutos. A distância média percorrida durante este teste foi de 2.956,88 \pm 90,69 metros $(n=16)$. Eissmann (15) observou que $80 \%$ dos árbitros que participaram do concurso da UEFA em 1995 percorreram, durante o teste de Cooper, uma distância de 2.900 a 3.200 metros. Embora a média dos árbitros paranaenses tenha sido de 2.956 metros, uma porcentagem menor dos árbitros paranaenses percorreram distâncias entre 2.900 e 3.200 metros. A variação da distância percorrida durante o teste de Cooper pode ser observada na Tabela 4. Os árbitros assistentes mostraram possuir uma capacidade aeróbica maior que a dos árbitros principais, sendo esta diferença estatisticamente significativa (Tabela 4). Como comentado anteriormente, já que o árbitro se desloca mais do que o árbitro assistente, deveria apresentar também uma capacidade aeróbica maior, para poder desempenhar a sua função de uma maneira satisfatória.

A cada 4,3 segundos o árbitro muda sua ação motora durante o jogo, realizando em média 1.268 atividades diferentes no transcorrer da partida (24). Para Catterall et al. (5) esta mudança ocorre a cada 6 segundos. Para uma atividade física desta magnitude, que deve ser sustentada por um período de 90 minutos, os árbitros deveriam apresentar uma capacidade aeróbica maior do que a apresentada neste estudo. Para Silva (26), como a maioria dos árbitros se apresenta para realizar os testes de aptidão física sem uma preparação física adequada, a concentração eventualmente elevada de lactato após os piques de 200 metros acaba prejudicando o desempenho durante o teste de Cooper. A má preparação física dos árbitros também foi observada por outros investigadores (26, 27) em estudos que tiveram como objetivo analisar 
as condições e fatores que podem causar reações de estresse psíquico nos árbitros de futebol. Foi concluído que uma preparação física inadequada é o fator mais estressante para o árbitro de futebol (27). O valor da média de idades dos árbitros deste estudo (34,5 anos; tabela 1) foi ligeiramente superior ao valor encontrado em árbitros da Federação Catarinense de Futebol - FCF, Brasil (32,6 anos) (28). Rontoyannis et al. (9) avaliaram 188 árbitros da região norte da Grécia com uma média de idades de 36,3 anos. Estes autores comentam que a idade dos árbitros quando comparada com a dos atletas de futebol é superior em cerca de 10 anos e que esta diferença é menor em outros esportes. Quando se compara a mediana de idade dos árbitros com a dos árbitros assistentes envolvidos neste estudo não se verificam diferenças estatisticamente significativas (Tabela 6). A estatura média $(177,8 \mathrm{~cm})$ dos árbitros analisados neste estudo era superior à observada nos árbitros da Federação Catarinense de Futebol $(174,4 \mathrm{~cm})$ (28) e semelhante à estatura dos árbitros gregos $(177,4 \mathrm{~cm})(9)$. Comparando a estatura dos árbitros com a de outros grupos populacionais nacionais, como os do levantamento realizado por Petroski (29) em indivíduos com idade entre 18 e 66 anos da região sul do Brasil, a diferença é de $3,3 \mathrm{~cm}$ a favor dos árbitros. A estatura média dos árbitros e dos árbitros assistentes deste estudo não se revelou significativamente diferente (Tabela 5).

A massa corporal média dos árbitros paranaenses é de $78,7 \mathrm{~kg}$. Este valor é ligeiramente superior à média $(76,4 \mathrm{~kg})$ dos árbitros catarinenses (28) e inferior à média (81,6 kg) dos árbitros gregos (9), porém $5,1 \mathrm{~kg}$ maior do que a dos homens da região sul do Brasil - 73,6 kg (29). Não foram encontradas diferenças estatisticamente significativas entre os valores médios destas variáveis quando se comparam os árbitros com os árbitros assistentes (Tabela 5). Com relação ao percentual de gordura, o valor médio $(15,9 \%)$ encontrado neste estudo é muito semelhante ao valor médio $(16,7 \%)$ encontrado nos árbitros gregos (9), porém muito menor do que o valor de Schwingel et al (30) num estudo realizado em 57 árbitros catarinenses $(20,7 \%)$. O percentual de gordura dos árbitros mostrou-se semelhante ao encontrado em homens da região sul do Brasil $(16,14 \%)$ (29). Os árbitros envolvidos neste estudo, apresenta- ram um percentual de gordura superior ao dos árbitros assistentes (Tabela 5). Os árbitros deveriam apresentar um percentual de gordura menor do que os árbitros assistentes, devido ao trabalho físico (por exemplo, a distância total percorrida) que realizam durante uma partida. Sabe-se que uma massa corpórea maior consome mais energia para ser deslocada, além de que o tecido adiposo serve como isolante térmico, colaborando para o aumento da desidratação, que pode vir a contribuir para o mau desempenho do árbitro durante uma partida.

A massa óssea encontrada neste estudo (13,3 kg) corresponde a $16,8 \%$ da massa corporal dos avaliados. Esta porcentagem é semelhante ao valor encontrado nos homens da região sul do Brasil $(16,42 \%)$ (29). A massa residual, por ser uma constante, é praticamente igual $(24,1 \%)$ em todos os grupos comparados. Quando analisamos o valor da massa muscular dos árbitros deste estudo (42,9\%), constatamos que esse valor em termos percentuais é muito semelhante ao encontrado nos homens da região sul do Brasil (43,0\%) (29). Não se observaram diferenças significativas para estas variáveis quando comparados os árbitros e os árbitros assistentes (Tabela 5). De acordo com as informações da tabela 6, neste estudo não foi possível observar qualquer correlação significativa entre as variáveis antropométricas e o desempenho nas provas de campo.

\section{CONCLUSÃO}

O tempo médio no teste de 50 metros dos árbitros do quadro da CBF pertencentes a FPF foi inferior ao tempo médio apresentado pelos árbitros assistentes destas mesmas entidades. O que contribui para uma melhor performance do árbitro durante uma partida de futebol, pois permite que o mesmo acompanhe as jogadas mais de perto.

O tempo médio gasto para percorrer os 200 metros pelos árbitros de futebol neste estudo foi de $28,85 \pm$ 1,57 segundos $(n=8)$, sendo o tempo médio do primeiro pique de $28,10 \pm 1,66$ e o do segundo de $29,59 \pm 1,90$. O aumento do tempo do segundo pique de 200 metros dos árbitros brasileiros evidencia a má preparação física destes, que não estão convenientemente preparados para esta prova.

Os valores da massa corporal e da estatura, quando comparados com os valores de outros estudos que 
envolvem árbitros, são ligeiramente maiores, porém o percentual de gordura é menor. Comparando-se os valores absolutos com os valores médios dos homens da região sul do Brasil, ou seja, da mesma região onde foi realizado este estudo, os árbitros apresentam maior estatura, maior massa corporal, maior massa óssea, maior massa muscular e menor massa de gordura. Sabendo que a idade dos árbitros do nosso estudo é superior à do homem de referência, pode-se considerar que os mesmos apresentam um bom perfil antropométrico e de composição corporal. Com base nos resultados observados neste estudo, pode concluir-se que a capacidade anaeróbica dos árbitros paranaenses, assim como a própria capacidade aeróbica, necessitam ser melhor desenvolvidas, principalmente nos árbitros principais. O diagnóstico aqui realizado é importante para a elaboração de programas de treinamento que contemplem estes aspectos por meio de exercícios específicos, que permitam a melhora da sua performance durante a partida de futebol e durante a realização da bateria de testes imposta pela FIFA. Um método que poderia ser utilizado para a melhoria da capacidade física dos árbitros, levando em consideração sua forma de deslocamento durante a partida e as características dos testes físicos, é o Fartlek e suas variações: fartlek natural, fartlek líder e fartlek especial (26), intercalado com trabalho de piques (25).

Os dados aqui apresentados servirão de referencial para árbitros e treinadores, que poderão comparar seus resultados com resultados de árbitros de duas grandes entidades (CBF e FPF), podendo assim estabelecer metas no programa de treinamento.

\section{Agradecimentos}

Agradecemos ao Prof. Ajunto de Bioquímica da UFPR, Aguinaldo José do Nascimento, por sua contribuição na análise estatística dos dados aqui apresentados.

\section{CORRESPONDÊNCIA}

\section{Alberto Inácio da Silva}

Unidade de Ensino Superior do Vale do Iguaçu, Uniguaçu

Rua Vitorino Polli, 286 - Jardim Adriana

CEP 83.408-408 - Colombo, Paraná

Brasil

albertoinacio@bol.com.br 


\section{REFERÊNCIAS}

1 Confederação Brasileira de Desportos (1978). Regras do futebol. Rio de Janeiro: Palestras Edições.

2 Antunes, P. (s/d.). Regras de futebol. São Paulo: Cia Brasileira Editora.

3 Duarte, O. (1997). Futebol: história e regras. São Paulo: Ed. Makron Books.

4 FIFA (1999). Regras do jogo. Zurich: Suíça.

5 Catterall, C.; Reilly, T.; Atkinson, G.; Coldwells, A. (1993). Analysis of the work rates and heart rates of association football referees. Br. J. Sp. Med., 27(3):193-196.

6 Johnston, L.; Mcnaughton, L. (1994). The physiological requirements of soccer refereeing. Aust J Sci Med Sport, 26 (3-4): 67-72.

7 Silva, A. I.; Rodriguez-Añez, C. R. (1999). Ações motoras do árbitro de futebol durante a partida. Treinamento Desportivo, 2(4):5-11. Londrina: Editora Treinamento Desportivo.

8 Silva, A. I.; Rodriguez-Añez, C. R. (2002). Ações motoras do árbitro assistente de futebol durante a partida. Revista Brasileira de Ciência e Movimento, 1 (10): 29-34. Brasilia: Editora Universa.

9 Rontoyannis, G.P.; Stalikas, A.; Sarros, G.; Vlastaris, A. (1998). Medical, morphological and funcional aspects of Greek football referees. Journal of Sports Medicine and Physical Fitness, 38:208-14.

10 Rodriguez-Añez, C. R.; Silva, A. I. (2001a). A freqüência cardíaca e a intensidade da atividade física do árbitro durante a partida de futebol. In, $16^{\circ}$ Congresso Internacional de Educação Física. Foz do Iguaçu, 143 - 148.

11 Rodriguez-Añez, C. R.; Silva, A. I. (2001b). A freqüência cardíaca e a intensidade da atividade física do árbitro assistente durante a partida de futebol. In, XIII Simpósio de Educação Física e Desportes do Sul do Brasil. Ponta Grossa, 154 - 159

12 Farinatti, P. T. V.; Monteiro, W. D. (1992). Fisiologia e avaliação funcional. Rio de Janeiro: Sprint.

13 Pollock, M. L.; Wilmore, J. H. (1993). Exercício na saúde e na doença. São Paulo: Medsi, $2^{\mathrm{a}}$ ed.

14 Astrand, P.; Rodahl, K. (1980). Tratado de fisiologia do exercício. Rio de Janeiro: Interamericana.

15 Eissmann, H. J. (1996). El árbitro de fútbol. Madrid: Editorial Gymnos.

16 Cuchiaro, A. L. (2000). Relação entre consumo/demanda energética, gordura corporal e estresse. Kinesis, (22)113-124

17 Harrison, G.G.; Buskirk, E.R.; Carter, J.E.L.; Johnston, F.E.; Lohman, T.G.; Pollock, M.L.; Roche, A.F.; Wilmore, J.H. (1991). Skinfold thicknesses and measurement technique. In. T.G. Lohman, A.F. Roche, R. Martorell (Eds.) Anthropometric standardization reference manual. Abridged Edition. Champaign, Illinois: Human Kinetics.

18 Callaway, C.W.; Chumlea, W.C.; Bouchard, C.; Himes, J.H.; Lohman, T.G.; Martin, A.D.; Mitchell, C.D.; Mueller, W.H.; Roche, A.F.; Seefeldt, V.D. (1991). Circumferences. In. T.G Lohman, A.F. Roche \& R. Martorell (Eds.) Anthropometric standardization reference manual. Abridged Edition. Champaign, Illinois: Human Kinetics.

19 Wilmore, J.H.; Frisancho, R.A.; Gordon, C.C.; Himes, J.H.; Martin, A.D.; Martorell, R.; Seefeldt, V.D. (1991). Body Breadth Equipment and Measurement Techniques. In. T.G Lohman, A.F. Roche \& R. Martorell, (Eds.) Anthropometric standardization reference manual. Abridged Edition. Champaign, Illinois: Human Kinetics.
20 Jackson, A.S. \& Pollock, M.L. (1978). Generalized equations for prediting body density of men. Br. J. Nutr. 40: 497-504.

21 Siri, W.E. (1961). Body composition from fluid space and density. In: J. Brozek \& A. Hanschel (Eds.), Techniques for measuring body composition. Washington, D.C.: National Academy of Science, 223-224.

22 De Rose, E.H.; Pigatto, E.; De Rose, R.C.F. (1984). Cineantropometria, educação física e treinamento desportivo. Rio de Janeiro: SEED/MEC.

23 Asami, T.; Togari, H.; Ohashi, J. (1988). Analysis of movement patterns of referees during soccer matches. In, $\mathrm{T}$. Reilly, A. Lees, K. Davids, W.J. Murphy (Eds.), Science and Football. London: E\& E N. Spon, 341-345.

24 Krustrup, P.; Bangsbo, J. (2001). Physiological demands of top-class soccer refereeing in relation to physical capacity: effect of intense intermittent exercise training. Journal of Sports Sciences, 19: 881-891.

25 Rebelo, A.; Silva, S.; Pereira, N.; Soares, J. (2002) Stress físico do árbitro de futebol no jogo. Revista Portuguesa de Ciências do Desporto, 5(2): 24-30.

26 Silva, A. I. (2002). La preparación física del árbitro de fútbol utilizando ejercicios de atletismo. Dissertação de Mestrado em Metodologia do Treinamento Desportivo. La Havana, Cuba: ISCF, Manuel Fajardo.

27 Samulski, D. M.; Noce, F.; Costa, E. G. (1999). Análise do estresse psicológico do árbitro: um estudo comparativo entre futebol e voleibol. Revista da APEF, 1 (14): 13 - 28. Londrina.

28 Velho, N. M.; Petroski, E. L.; Schwingel, A. C. (1998) Índice de massa corporal (IMC) em árbitros da FCF (Resumo). In, Anais do XXI Simpósio Internacional de Ciências do Esporte. São Paulo, 96.

29 Petroski, E.L. (1995). Desenvolvimento e validação de equações generalizadas para a estimativa da densidade corporal em adultos. (Tese de Doutorado). Doutorado em Educação Física, UFSM, Santa Maria, RS.

30 Schwingel, A. C.; Michels, G.; Petroski, E. L.; Velho, M. N. (1998). Análise comparativa da composição corporal de jogadores e árbitros de futebol de campo (Resumo). In, Anais do XXI Simpósio Internacional de Ciências do Esporte. São Paulo, 77 